\title{
Serial Topography Analysis After Acute Unilateral Diffuse Lamellar Keratitis
}

Jay Lytle, OD, FAAO

Private practice, Worthington, Ohio

\begin{abstract}
INTRODUCTION
Diffuse lamellar keratitis is a sterile inflammatory response along the lamellar interface in corneal refractive surgeries such as laser in-situ keratomileusis (LASIK) and small incision lenticule extraction (SMILE). It usually presents within one to seven days after the procedure, although late-onset cases have been observed..$^{1-3}$
\end{abstract}

Diffuse lamellar keratitis typically presents with fine white interface deposits beginning in the periphery and spreading diffusely toward the center of the cornea. It has been suggested that diffuse lamellar keratitis may represent a classic acute polymorphonuclear inflammatory reaction triggered by antigens or toxins introduced to the flap interface during surgery. ${ }^{4,5}$ Potential sources include ocular trauma, epithelial defects, Meibomian gland secretions, sterilized bacterial cell wall endotoxins, oil residues on autoclaved surgical equipment (i.e., microkeratomes and cannulas), balanced salt solution, environmental agents, and powdered surgical gloves. ${ }^{2,4,5}$ Some of the earliest reports of diffuse lamellar keratitis were published in 1998, and the incidence of this condition has been estimated to be 1 in 500 cases. $^{2}$ It is estimated that approximately $70 \%$ of cases are unilateral. ${ }^{2}$

In the early stages, patients are usually asymptomatic. In the later stages, symptoms can include blurred vision, foreign body sensation, and light sensitivity, and in some cases the release of inflammatory mediators from coalesced white blood cells can cause stromal necrosis and a permanent reduction in best corrected visual acuity due to corneal scarring. ${ }^{6}$ Important differential diagnoses include pressure-induced steroid keratitis (PISK), central toxic keratopathy (CTK), benign interface debris, and bacterial keratitis. . $^{1,4,5,7}$

All stages of diffuse lamellar keratitis require topical steroids and success has been observed with a rapid taper ${ }^{4,5}$ Lifting the flap and irrigating the interface is recommended once the condition reaches stage three, to avoid conversion to stage four. ${ }^{7}$ Oral doxycycline and $10 \%$ sodium citrate have also been shown to be clinically effective in the treatment of diffuse lamellar keratitis. ${ }^{10}$

The case presented here provides a quantitative summary of the topographical stabilization that occurs after the resolution of diffuse lamellar keratitis on slit lamp examination and provides clinicians with a timeframe with which to educate their patients on their potential refractive resolution.

CASE REPORT

A 53-year-old man presented to TLC Laser Eye Center (Dublin, OH, USA) with acute unilateral diffuse lamellar keratitis of the right eye 11 days after receiving bilateral wavefront optimized femtosecond-assisted hyperopic LASIK. 
The patient's pre-operative dry manifest refraction was $+3.50-0.50 \times 053$ in the right eye and $+3.75-0.50 \times 147$ in the left eye. The patient's medical history was positive for hypertension, anxiety, and hypercholesterolemia, and he denied any pertinent ocular history. He reported taking atenolol, Prozac, and an unknown statin medication to treat the aforementioned systemic conditions. He had no known medication allergies.

During surgery, sterile balanced salt solution (BSS) was used for normal intra-operative irrigation. All surgical tools were autoclaved using a STATIM cassette autoclave unit, and left to dry prior to use. The surgeon and staff wore nonpowdered surgical gloves. The patient's corneal flaps were created using an Alcon FS200 femtosecond laser. The flap diameter was $8.8 \mathrm{~mm}$ with a 70-degree side-cut and a 120-micron thickness in each eye. The suction time was 49 seconds for the right eye and 43 seconds for the left eye, with actual flap formation lasting approximately eight seconds in both eyes. The patient's ablation treatment was performed using an Alcon EX500 excimer laser. The programmed treatment, using the surgeon's nomogram adjustment, was +3.50-0.25x053 OD and +3.75-0.25x147 OS. The ablation lasted 13 seconds for the right eye and 14 seconds for the left eye. The surgery was performed without complications.

The patient returned for his day 1 post-operative visit and denied any blurred vision or ocular discomfort. He reported taking gatifloxacin four times daily in both eyes, prednisolone acetate $1 \%$ every two hours, while awake, in both eyes, and preservative-free artificial tears hourly, while awake, in both eyes, as prescribed. The patient's uncorrected distance visual acuity was 20/25 in the right eye and 20/25 in the left eye. Slit lamp examination revealed no pathology of the lid or adnexa in either eye. The palpebral and bulbar conjunctiva were clear in both eyes, with no signs of edema or injection. The patient's corneas were clear, with both corneal flaps in proper position, having smooth edges, no striae, no haze, and no interface opacities. The anterior chamber was deep and quiet, with no signs of cells or flare in either eye. The patient was advised to continue his post-operative eye drops as directed. Due to the patient's schedule, he was unable to return for his second post-operative visit between days 5 and 7, as recommended. Instead, he scheduled his second follow-up visit on day 11. The patient was advised to contact his surgical center immediately if he noticed any ocular discomfort or worsening of his vision.

Diffuse lamellar keratitis presented at the day 11 post-operative visit, when the patient's uncorrected visual acuity was $20 / 50^{-2}$ in the right eye (pinhole $20 / 25^{-1}$ ) and $20 / 20^{-2}$ in the left eye. The patient reported mild constant blurred vision and mild light sensitivity of the right eye. He mentioned sleeping with his fan on at night and wondered if the blur may have been caused by ocular dryness. Upon further questioning, the patient stated that the asymmetry in his visual acuity and his increased light sensitivity began six days prior (five days after his surgery); however, he did not feel his symptoms were significant enough to contact his surgical center. The patient's dry manifest refraction was $+1.00-0.50 \times 146\left(20 / 30^{+1}\right)$ in the right eye and $+0.25-0.25 \times 168\left(20 / 20^{-1}\right)$ in the left eye. He was using prednisolone acetate $1 \%$ four times per day in both eyes and his right cornea showed central microstriae, grade 2+ diffuse stromal haze at the depth of the flap interface with a slight confluence centrally, and grade 2+ fine white opacities scattered diffusely along the flap interface. The flap edge was smooth, with no signs of epithelial ingrowth. The patient's left cornea was clear, with its flap in proper position, with no striae, no haze, and no interface opacities. Sodium fluorescein revealed no epithelial defects, and conjunctival hyperemia was not present in either eye. The patient's intraocular pressure using Tonopen applanation tonometry on the central cornea was $13 \mathrm{mmHg}$ in the right eye and $12 \mathrm{mmHg}$ in the left eye, measured at 10:20 am. The patient was diagnosed with diffuse lamellar keratitis of the right eye and treatment with prednisolone acetate $1 \%$ ophthalmic suspension (one drop every hour, while awake) was initiated in the right eye.

Diffuse lamellar keratitis improved with a gradual steroid taper (hourly for one week, every other hour for one week, four times daily for one week, once daily for one week), and reached final resolution at the day 32 post-operative visit (21 days after the initial diagnosis). One week later (day 39), the patient denied any further symptoms of blur or light sensitivity. At each post-operative visit, the patient's central and peripheral intraocular pressures were monitored to rule out PISK, and were found to be within the normal range. After the condition was no longer visible on slit lamp examination, the patient was followed regularly to monitor his refractive error and Pentacam tomography (Oculus, Wetzlar, Germany). Initially, the axial / sagittal curvature maps of the right eye showed local areas of steepening and flattening adjacent to the central confluence of diffuse lamellar keratitis. The left eye showed a relatively uniform post-LASIK hyperopic ablation. On day 88, the patient remained asymptomatic and both of his eyes showed a relatively uniform post-LASIK hyperopic ablation, with only a few areas of residual flattening of the right eye centrally. The patient's final uncorrected distance visual acuity was $20 / 25^{+1}$ in the right eye and $20 / 15^{-2}$ in the left eye, with a dry manifest refraction of +0.50 sphere $(20 / 20)$ and $+0.50-0.50 \times 162(20 / 15)$, respectively. 
TABLE 1: Axial/sagittal curvature maps (Pentacam), pachymetry maps (Pentacam), and associated clinical findings

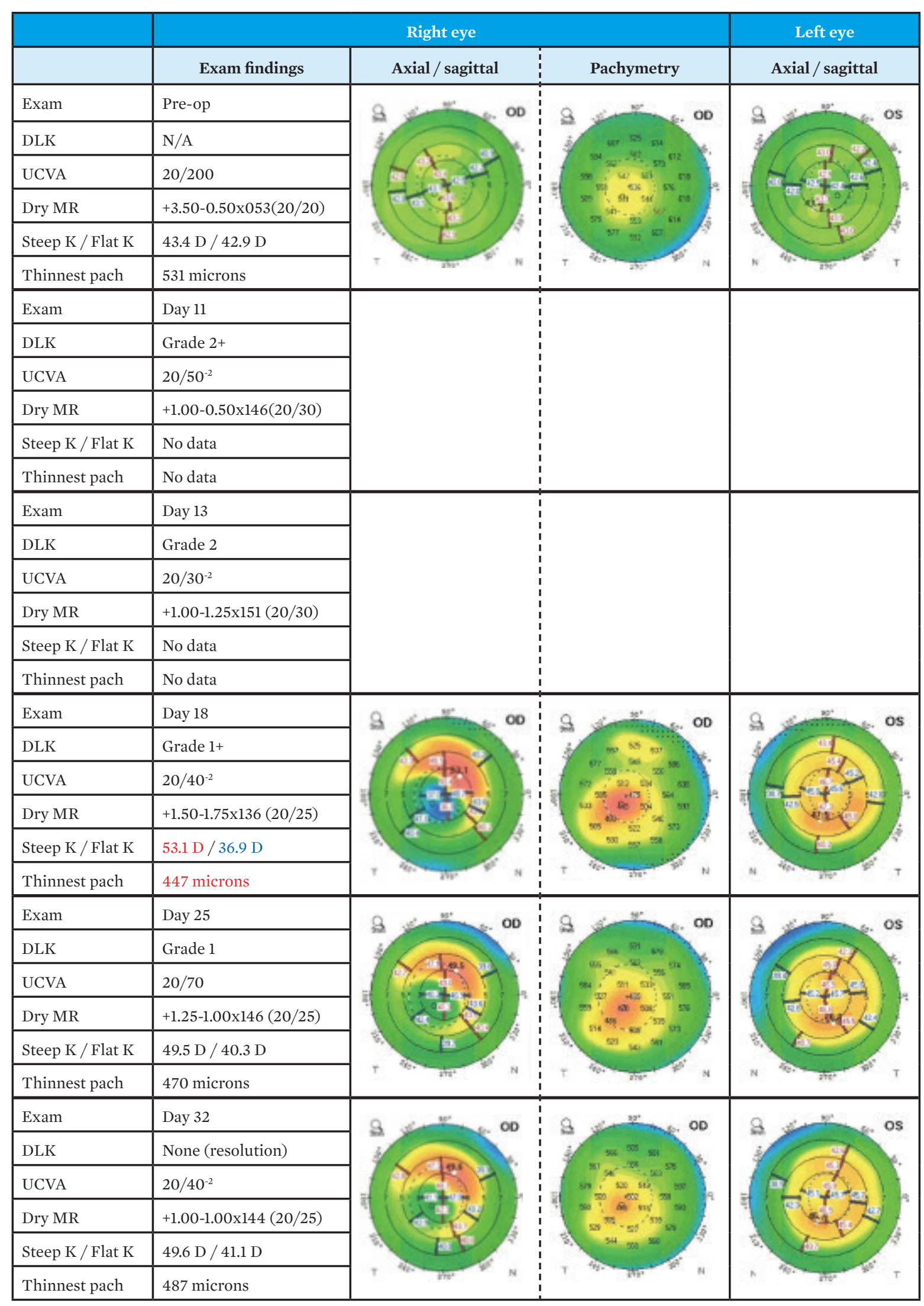


TABLE 1: continued

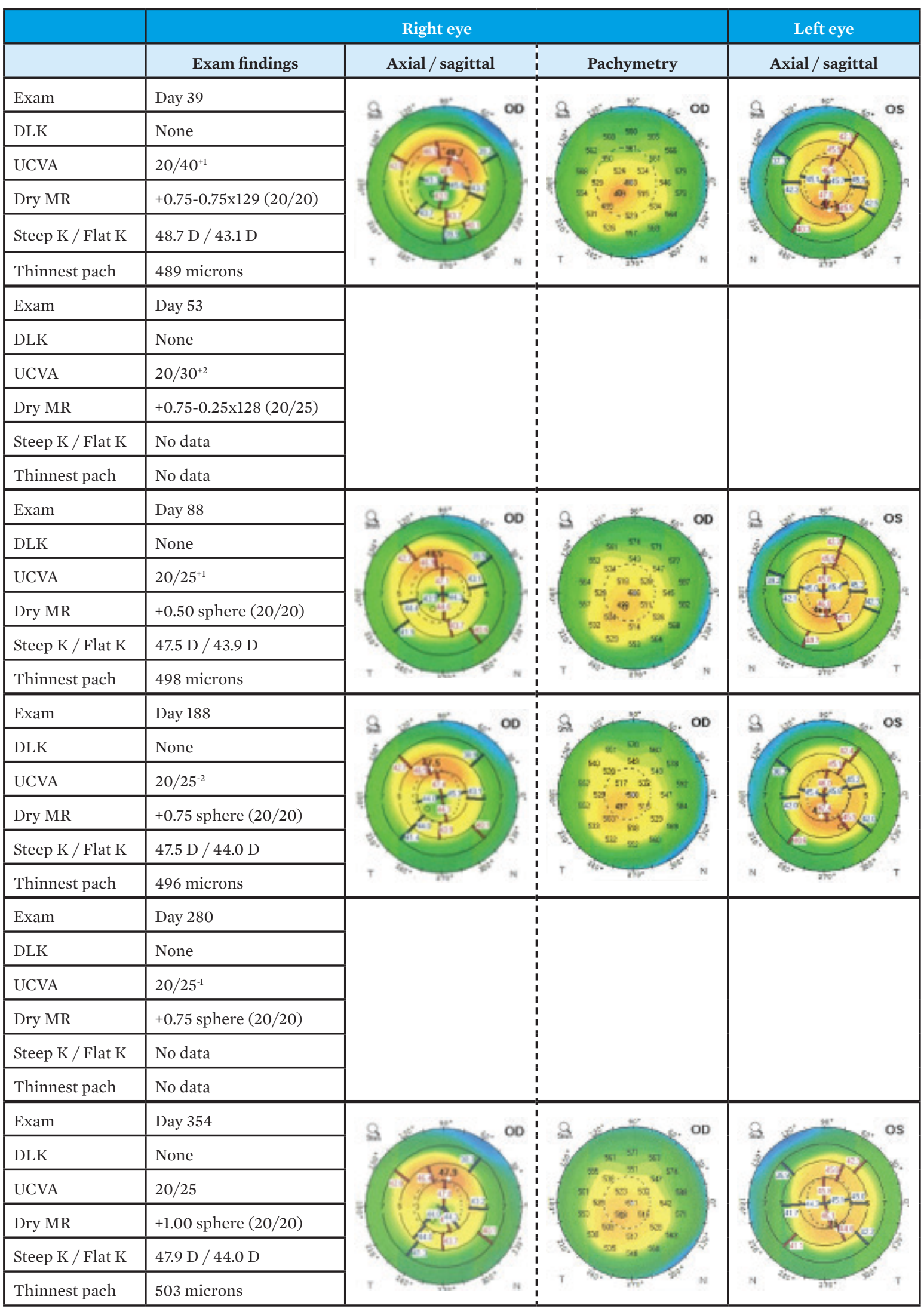


Figure 1: Topographic stabilization

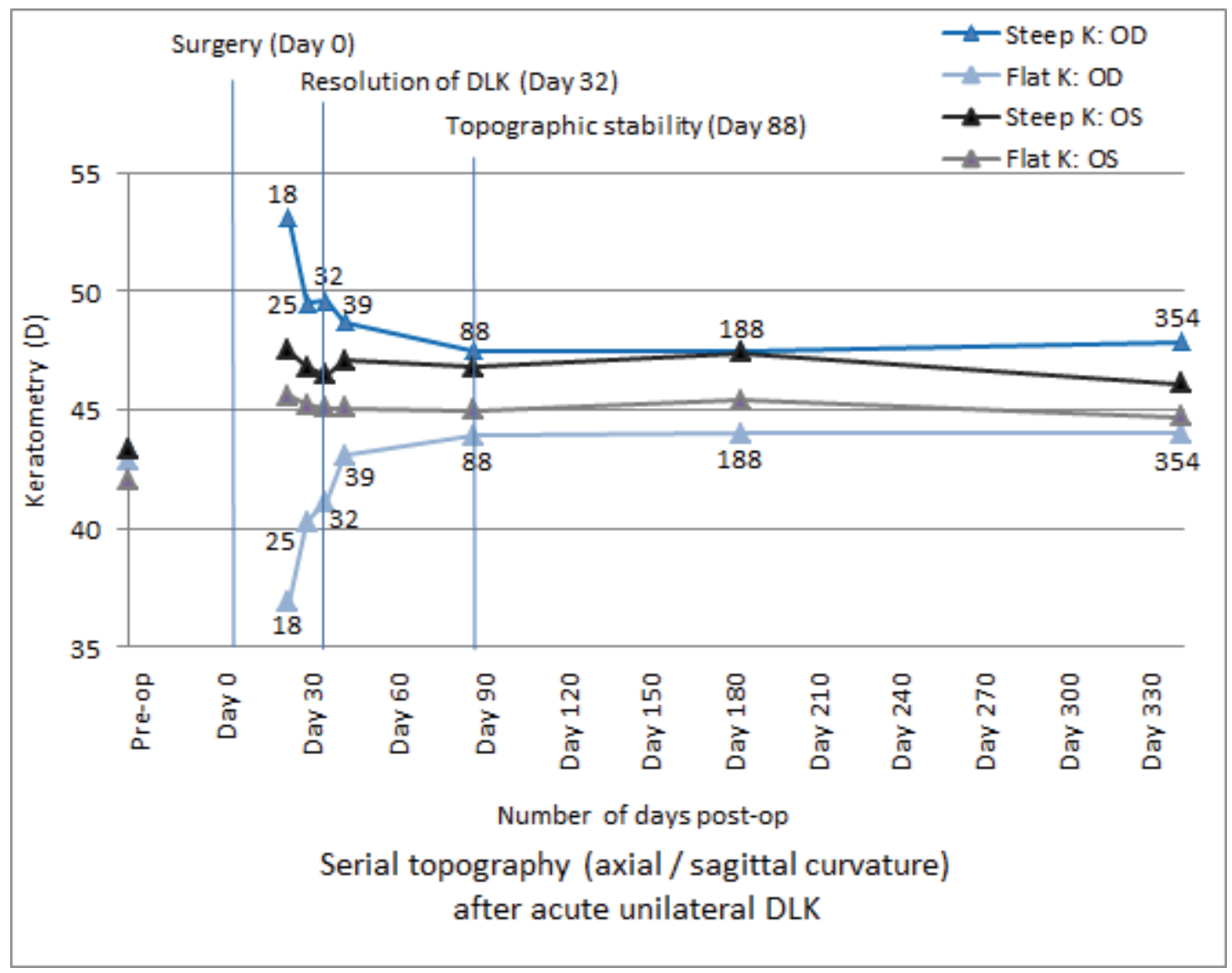

\section{DISCUSSION}

From the time this patient's diffuse lamellar keratitis resolved on day 32, the disparity between the steepest and flattest points on his keratometry lessened and the patient's refractive error became more spherical. By day 88, the patient transitioned from one diopter of oblique astigmatism to completely spherical on manifest refraction, and from a disparity of 8.5 diopters on his steep and flat keratometry readings (49.6-41.1) to a disparity of 3.6 diopters (47.5-43.9). At the patient's three remaining post-operative visits (day 188, day 280, day 354), no significant changes were observed in his topography.

The patient's Pentacam pachymetry maps revealed corneal thinning adjacent to the diffuse lamellar keratitis with thinnest local points of 531 microns (pre-op baseline), 447 microns (day 18), 470 microns (day 25), 487 microns (day 32), 489 microns (day 39), 498 microns (day 88), 496 microns (day 188), and 503 microns (day 354). As noted with the axial / sagittal topography, the pachymetry findings stabilized by day 88 .

Therefore, it appeared that all the clinically significant changes associated with this patient's diffuse lamellar keratitis took place within the first three months after the initial diagnosis. Resolution of the patient's clinical signs and symptoms was observed in the first month, while topographical and refractive resolution was observed by the third month. Similar case reports in patients with grade four diffuse lamellar keratitis noted resolution in topography and refractive error after 6 to 12 months. ${ }^{10,11}$

Based on the patient's presentation in this case study, the severity of the diffuse lamellar keratitis at the initial presentation was likely to have been between stages two and three. The response to the prescribed treatment prevented the need for flap lift and irrigation. The hyperopic regression in the right eye from day $88(+0.50$ sphere $)$ to day 354 $(+1.00$ sphere $)$ may have been caused by post-LASIK hyperopic regression $(+3.50-0.25 \mathrm{x} 053$ treated $)$, and was not necessarily associated with the diffuse lamellar keratitis. 


\section{CONCLUSION}

This case study illustrates that the coalescence of white blood cells along a flap interface can cause local areas of keratometric change, often inducing hyperopia and irregular astigmatism. These changes associated with diffuse lamellar keratitis show resolution even after clinical signs have dissipated. In this case, refractive stability was observed two months after diffuse lamellar keratitis was no longer visible on slit lamp examination.

\section{REFERENCES}

1. Bowling, B. Kanski’s Clinical Ophthalmology: A Systematic Approach, $8^{\text {th }}$ ed. Amsterdam: Elsevier, 2016.

2. Probst LE. Complex Cases with LASIK: Advanced Techniques and Complication Management. Thorofare, NJ: SLACK Inc., 2000.

3. Morales P, Camos-Carreras A, Spencer F, et al. Late-onset diffuse lamellar keratitis 15 years after laser in situ keratomileusis. Can J Ophthalmol 2017;52:3, 91-3.

4. Probst LE, Tsai JH. Ophthalmology Clinical and Surgical Principles. Thorofare, NJ: SLACK Inc., 2012.

5. Gimbel HV, Penno EA. LASIK Complications: Prevention and Management. Thorofare, NJ: SLACK Inc., 1999.

6. Boyd BF. LASIK and Beyond LASIK: Wavefront Analysis and Customized Ablation. $1^{\text {st }}$ ed. El Dorado, Panama: Highlights of Ophthalmology International, 2001.

7. Randleman JB, Shah RD. LASIK interface complications: etiology, management, \& outcomes. J Refract Surg 2012;28:575-86.
8. Davidson RS, Brandt JD, Mannis MJ. Intraocular pressure-induced interlamellar keratitis after LASIK surgery. J Glaucoma 2003;12:23-6

9. Sonmez B, Maloney RK. Central toxic keratopathy: description of a syndrome in laser refractive surgery. Am J Ophthalmol 2007;143:420-7.

10. Balestrazzi A, Balestrazzi A, Giannico M, I, et al. Diagnosis, clinical trend, and treatment of diffuse lamellar keratitis after femtosecond laser-assisted in situ keratomileusis: a case report. Ophthalmology 2018;9:457-64.

11. Michieletto P, Balestrazzi A, Balestrazzi A, et al. Stage 4 diffuse lamellar keratitis after laser in situ keratomileusis Clinical, topographical, and pachymetry resolution 5 years later. J Cataract Refract Surg 2006;32(2):353-6. 